\title{
Effect of Follicular Fluid Lactoferrin Level on Oocytes Quality and Pregnancy Rate in Intracytoplasmic Sperm Injection Cycles
}

\section{Mohamed Hussein Mostafa ${ }^{1}$, Malames Mahmoud Faisal2 ${ }^{*}$, Noha Refaat Mohamed ${ }^{1}$, Filsan Houssein Idle ${ }^{1}$}

\author{
${ }^{1}$ Department of Obstetrics and Gynecology, Faculty of Medicine, Ain Shams University, Cairo, Egypt \\ ${ }^{2}$ Department of Clinical Pathology, Faculty of Medicine, Ain Shams University, Cairo, Egypt \\ Email: ^misofaisal@yahoo.co.uk, *misofaisal@med.asu.edu.eg
}

How to cite this paper: Mostafa, M.H., Faisal, M.M., Mohamed, N.R. and Idle, F.H. (2019) Effect of Follicular Fluid Lactoferrin Level on Oocytes Quality and Pregnancy Rate in Intracytoplasmic Sperm Injection Cycles. Open Journal of Obstetrics and Gynecology, 9, 745-754. https://doi.org/10.4236/ojog.2019.96074

Received: April 28, 2019

Accepted: May 28, 2019

Published: May 31, 2019

Copyright $\odot 2019$ by author(s) and Scientific Research Publishing Inc. This work is licensed under the Creative Commons Attribution International License (CC BY 4.0).

http://creativecommons.org/licenses/by/4.0/

\begin{abstract}
Assessment of oocyte quality to avoid overproduction of embryos is now considered an important goal in ICSI cycles. Although in classic Intra Cytoplasmic Sperm Injection (ICSI) cycles selection is mainly done for embryos and not for follicles. Follicular fluid and its contents representing the oocyte environment are now gaining more attention because not only of its crucial influence on oocyte developments but also due to easy isolation of follicular fluid with every case of ICSI. By its aspiration during ovum pick up (OPU). substances isolated from follicular fluid to assess oocyte quality were in the form of: hormones like, Follicle Stimulating Hormone (FSH), Luteinizing Hormone (LH), Anti-mullerian Hormon (AMH), Growth Hormone (GH) etc., growth factors like Insulin Like Growth Factor (ILGF), proteins and amino acids like Lactoferrin (LF) which is an iron-binding glycoprotein that was detected in follicular fluid and was thought to be related to good oocyte quality when present in high concentration. Objectives: The aim of this study was to detect the possible effect of follicular fluid lactoferrin on oocyte quality and hence pregnancy rate in ICSI cycles. Methods: Follicular fluid was obtained from 64 patients undergoing Intra-cytoplasmic sperm injection (ICSI) procedure in Ain Shams University Maternity Hospital. Follicular fluid (FF) was collected at the time of oocyte harvesting. The lactoferrin concentration in FF was assayed by ELISA. Results: The mean LF concentration in follicular fluid of the positive biochemical pregnancy group $(0.63 \pm 0.17 \mathrm{ng} / \mathrm{mL})$ was not significantly higher than that in the negative biochemical pregnancy $(0.61$ $\pm 0.16 \mathrm{ng} / \mathrm{mL}$ ). A positive correlation between a number of mature oocytes and lactoferrin concentration was not found (r.101). Conclusion: Lactoferrin level in follicular fluid does not correlate with oocytes quality or pregnancy rate.
\end{abstract}




\section{Keywords}

Lactoferrin, Infertility, Follicular Fluid, Oocyte Quality

\section{Introduction}

Oocyte quality estimation is a major goal in human in-vitro fertilization to limit embryos overproduction. The process of oocytes equality determination started from oocyte morphology and progressed towards genetic and biochemical factors detection [1].

Follicular fluid (FF), which consists of products of components that crossed the blood follicular barrier as well as products of the secretory activity of granulosa and theca cells, represents the oocyte microenvironment so it plays a vital role in oocyte development and maturation. FF constituents are considered to determine oocyte quality and subsequently embryo quality [2].

Lactoferrin LF which is an iron-binding glycoprotein isolated from milk [3], [4]. Lactoferrin has a molecular weight of about $80 \mathrm{kDa}$, and has high affinity for iron. The molecular structure and amino acid sequence of human lactoferrin were first described in 1984. Lactoferrin was classified as a member of the transferrin family, due to its $60 \%$ sequence similarity with serum transferring [5]. Lactoferrin has 3 different isoforms: Lactoferrin- $\alpha$, lactoferrin- $\beta$ and lactoferrin- $\gamma[6]$.

Follicular fluid Lactoferrin content comes through crossing the blood follicular barrier from the serum, and also produced by theca cells [7].

According to a prior study, interleukin-8 (IL-8) was found to increase in follicular fluid, and interleukin-8 may have an influence on follicular maturation [8] [9] [10]. Neutrophil production of lactoferrin is well-known [11], and the relation of lactoferrin production with IL-8 was reported [12], which indicates that LF may play a major role in the reproductive procedure [13].

Lactoferrin may influence granulosa cells to regulate the release of cytokines like IL-8 and TNF. It was newly reported that gamma-aminobutyric Acid-B2 receptor was down-regulated by lactoferrin, and LF may modify the level of intracellular cyclic adenosine 3=:5= monophosphate (cAMP) [14]. Oocyte maturation was noticed to be affected by the decrease of cAMP level in the oocyte after ovulation [15]. Lactoferrin may be included in that process by the control of the cAMP level. As good ovarian follicle is considered to produce good embryo, lactoferrin in the follicular fluid may be one of the biological makers to select the embryos at the time of embryo transplantation. [16]

This study aimed to detect the possible effect of follicular fluid lactoferrin on oocyte quality and hence pregnancy rate in ICSI cycles.

\section{Materials and Methods}

This study is a cross sectional study that was conducted from May 2018 to De- 
cember 2018. By using PASS program $11^{\text {th }}$ release, setting alpha error at $5 \%$, power of test at $90 \%$ and according to results of previous similar study (Yanaihara et al., 2007), the needed sample was 64 cases undergoing ICSI. [13].

Following study approval by the institutional review boards and ethics committee of Ain Shams University Maternity Hospital, An informed consent was obtained from all participants.

The age, BMI of cases was recorded as well as hormonal profile results, duration and cause of infertility. The indication for ICSI was either tubal factor diagnosed by hysterosalpingography or male factor where sperm concentration and motility examined according to WHO before ICSI or unexplained infertility diagnosed by excluding all causes of infertility. ovarian factors of infertility were excluded.

Inclusion criteria:

- Women age range from 20 - 40 years old.

- BMI range $20-35 \mathrm{~kg} / \mathrm{m}^{2}$.

- Infertility cause is either: tubal, male or unexplained. Exclusion criteria:

- Patients age $<20$ years or $>40$ years.

- $\mathrm{BMI}>35 \mathrm{~kg} / \mathrm{m}^{2}$.

- Women with Ovarian causes of infertility for e.g.; PCOS or decreased ovarian reserve.

After patients selection, full history, general, abdominal examination and hormonal profile were done. Controlled ovarian hyperstimulation using flexible antagonist technique was started at the second day of menstrual cycle with recombinant human $\mathrm{rFSH}$. The daily individualized (rFSH) dose (Gonapure 150 IU) ranged between 150 and $450 \mathrm{IU}$, according to BMI, age of the women, and the expected ovarian response. The dose was adjusted according to patient's response which was assessed by transvaginal ultrasound folliculometry which was done on day 6 . And after that it was done day after day till leading follicle size reaches $18 \mathrm{~mm}$ or more. GnRH antagonist (Cetrotide $0.25 \mathrm{mg}$ ) subcutaneous daily dose was started when the leading follicle size is $>$ or equal to $14 \mathrm{~mm}$ seen in folliculometry. Intramuscular human chorionic gonadotrophin (hCG) $(10,000$ IU) single dose was administered when folliculometry revealed the average diameter of 3 preovulatory follicles had approached $18-20 \mathrm{~mm}$.

Oocyte retrieval was performed under general anesthesia (intravenous administration of Propofol) 36 hours following hCG administration using vaginal ultrasound guided single lumen needle. The collected oocytes were incubated with $5 \% \mathrm{CO}_{2}$ at $37^{\circ} \mathrm{C}$ for $3-4$ hours, and then were used for ICSI. Follicular fluid of only mature follicles $(>17 \mathrm{~mm})$ aspirated from each patient was collected in a sterile tube, separated from the cumulus-oocyte complex and then centrifuged at $500 \times \mathrm{g}$ for $5 \mathrm{~min}$ and kept frozen at $-70^{\circ} \mathrm{C}$ until analysis, ICSI was performed and. Oocyte maturity at time of insemination was evaluated and graded according to Lin et al. [17]. 
A single motile sperm was injected into oocytes. After 24 hours, oocytes were separated from surrounding granulosa cells. Fertilization was based on the basis of two distinct pronuclei and two polar corpuscles. Embryos quality was evaluated into G1, G2, G3, G4 and G5 according to Gardner's et al. [18].

A maximum of three embryos were transferred at $4-8$ cell stages under ultrasound guidance. Chemical pregnancy was evaluated by $\beta$-hCG test 14 days after embryo transfer. The lactoferrin level of every respective follicular fluid was measured using a second-generation ELISA

\section{Lactoferrin concentration measurement of follicular fluid:}

The quantitative determination of Follicular Lactoferrin was done using the commercially available ELISA kit supplied by Wuhan EIAab Science ( $3^{\text {rd }}$ Floor, Building A4, Biopark, Optics Valley, Wuhan, CHINA). The micro titer plate provided in this kit has been precoated with an antibody specific to LTF/LF. Standards or samples were then added to the appropriate microliter plate wells with a biotin-conjugated polyclonal antibody preparation specific for LTF/LF. Next, Avidin conjugated to Horseradish Peroxidase (HRP) was added to each microplate well and incubated. Then a 3,3',5,5'-tetramethylbenzidine (TMB) substrate solution was added to each well. Only those wells that contain LTF/LF, biotin-conjugated antibody and enzyme-conjugated Avidin exhibited a change in color. The enzyme-substrate reaction was terminated by the addition of a Sulphur acid solution and the color change in the samples was then determined by comparing the Optical Density of the samples to the standard curve.

\section{Statistical Analysis}

Univariate analysis was accomplished for each variable. Continuous parametric data are presented as mean \pm standard deviation (SD) and categorical variables as numbers and percentages. The Mann-Whitney test, Student $\mathrm{T}$ and Spearman correlations were used to compare lactoferrin levels according to quantitative variables, Chi-Square test and Fisher's exact test were used to examine the relationship between two qualitative variables when the expected count is less than 5 in more than $20 \%$ of cells.

The ability of follicular fluid lactoferrin level to predict the biochemical pregnancy outcome was determined by constructing the Receiving Operator Curve (ROC) curve and calculating the area under the curve (AUC) with $95 \%$ confidence intervals (CI). The sensitivity and specificity for the optimal cut-off were calculated. Statistical tests were performed using the R (version 2.15.2) software. Results were considered significant when $\mathrm{p} \leq 0.05$.

\section{Results}

Patients' characteristics are detailed in (Table 1). age of the participants was 30.7 \pm 4.3 years (range: 20 to 40 years) and the BMI was $28.9 \pm 2.4 \mathrm{~kg} / \mathrm{m}^{2}$ (range: 23 to $33 \mathrm{~kg} / \mathrm{m}^{2}$ ). The duration of infertility was $3.7 \pm 2.5$ years (range 1 to 13 years). It was the first ICSI cycle For 39 cases (61\% of the participants). Cause of infertility were tubal factor ( 45 cases $70.3 \%$ ), unexplained (11 cases $17.2 \%$ ), and male factor ( 8 cases $12.5 \%)$, 
Table 1. Demographic characteristics of the whole study population.

\begin{tabular}{cccccc}
\hline Variable & Mean & \pm SD & Minimum & Maximum & Median (IQR) \\
\hline Age (years) & 30.7 & 4.3 & 20.0 & 40.0 & \\
BMI $\left(\mathrm{Kg} / \mathrm{m}^{2}\right)$ & 28.9 & 2.4 & 23 & 33.3 & \\
Duration of infertility (years) & 3.7 & 2.5 & 1.0 & 13.0 & \\
Cause of infertility & & & & & \\
$\quad$ Tubal & 45 & $70.3 \%$ & & \\
Un-explained & 11 & $17.2 \%$ & & \\
Male & 8 & $12.5 \%$ & &
\end{tabular}

The ovarian stimulation length ranged between 8 to 12 days (9.7 \pm 0.8 days), Number of oocytes retrieved from each patient ranged from 0 to 30 oocyte with a mean of ( $12.1 \pm 6.3$ oocytes) while number of mature oocytes was $7 \pm 4$ oocytes (Table 2).

There was a non-significant difference between both groups of positive biochemical pregnancy (44 cases $68.7 \%$ ) and negative biochemical pregnancy (20 cases $31.3 \%$ ) regarding; age, BMI, infertility duration, infertility cause, number of retrieved, mature, fertilized oocytes or number of transferred embryos (Table 3).

The results also show that there was no statistically significant difference between women with positive and negative biochemical pregnancy regarding mean concentration level of lactoferrin in follicular fluid $(0.6 \pm 0.1 \mathrm{ng} / \mathrm{ml})$ versus $(1.5$ $\pm 6.0 \mathrm{ng} / \mathrm{ml}$ ) respectively, $\mathrm{p}=0.636$ (Table 3 ).

Using ROC curve, it was shown that Level of lactoferrin in follicular fluid could not be used to discriminate between females with negative and positive biochemical pregnancy cases $(\mathrm{P}=0.64$, sensitivity and specificity $45 \%$ and $65.12 \%$ respectively) (Table 4 \& Figure 1 ).

Table 5 shows no significant correlations between number of retrieved, mature or fertilized oocytes with Level of lactoferrin in follicular fluid.

\section{Discussion}

Follicular fluid (FF) components were considered to play an important role in the determination of oocyte quality, fertilization and embryo quality being the oocyte microenvironment so, study of FF components was considered as noninvasive methods for prediction of oocyte quality and hence embryo quality which is a main factor for ICSI process success [19]. Lactoferrin (LF) is a transforming growth factor and its expression was first described in in the 2-4-cell fertilized mouse embryo and continued until the blastocyst stage of development; the expression nearly disappeared in the hatched blastocyst. These findings might indicate that lactoferrin has a unique paracrine role in the development of the trophectoderm lineage during preimplantation development. [20]. Neutrophil production of LF is also well-known [11], and the correlation of LF production with IL-8 was recorded in previous researches [12]. 
Table 2. Description of the induction process, number of preovulatory follicles, retrieved, mature and fertilized oocytes, number of transferred embryos and lactoferrin concentration in follicular fluid.

\begin{tabular}{|c|c|c|c|c|c|}
\hline Variable & Mean & $\pm \mathrm{SD}$ & Minimum & Maximum & Median (IQR)* \\
\hline Number of follicles (16-18 mm) & 8.3 & 3.1 & 2.0 & 17.0 & $8(6-10.5)$ \\
\hline $\begin{array}{l}\text { Level of lactoferrin in follicular fluid } \\
\qquad(\mathrm{ng} / \mathrm{ml})\end{array}$ & $1.2 \mathrm{ng} / \mathrm{ml}$ & 5.0 & $0.2 \mathrm{ng} / \mathrm{ml}$ & $40.0 \mathrm{ng} / \mathrm{ml}$ & $0.7(0.5-0.7)$ \\
\hline Number of retrieved oocytes & 12.1 & 6.3 & 0.0 & 30.0 & $12(8-16)$ \\
\hline Number of mature oocytes & 7.0 & 4.0 & 0.0 & 20.0 & $7(4-9)$ \\
\hline Number of fertilized oocytes & 5.1 & 2.6 & 0.0 & 13.0 & $5(4-6.5)$ \\
\hline Number of transferred embryo. & 2.3 & 0.9 & 0.0 & 3.0 & $2(2-3)$ \\
\hline
\end{tabular}

${ }^{\star}$ Inter quartile range.

Table 3. Comparison between females with positive and negative biochemical pregnancy.

\begin{tabular}{|c|c|c|c|c|c|c|c|c|}
\hline & \multicolumn{6}{|c|}{ Biochemical pregnancy } & \multirow{3}{*}{$\mathbf{P}$} & \multirow{3}{*}{ Significant } \\
\hline & \multicolumn{3}{|c|}{ Negative } & \multicolumn{3}{|c|}{ Positive } & & \\
\hline & mean & $\pm \mathrm{SD}$ & Median & mean & $\pm \mathrm{SD}$ & $\operatorname{median}^{\star *}$ & & \\
\hline Age (years) & 30.8 & 4.6 & & 30.7 & 4.0 & & $0.913^{*}$ & NS \\
\hline BMI $\left(\mathrm{kg} / \mathrm{m}^{2}\right)$ & 29.1 & 2.1 & & 28.6 & 3.0 & & $0.404^{*}$ & NS \\
\hline Duration of infertility (years) & 4.1 & 2.7 & $3(2-6)$ & 3.0 & 1.8 & $3(1-5)$ & $0.14^{*}$ & NS \\
\hline \multicolumn{9}{|l|}{ Cause of infertility } \\
\hline Tubal & 27 & $61.4 \%$ & & 18 & $90.0 \%$ & & $0.06^{* * *}$ & NS \\
\hline Male & 6 & $13.6 \%$ & & 2 & $10.0 \%$ & & & NS \\
\hline Un-explained & 11 & $25 \%$ & & 0 & $.0 \%$ & & & NS \\
\hline Number of follicles (16-18 mm) & 7.9 & 3.2 & $8(5-10)$ & 9.0 & 2.7 & $9.5(6-12)$ & $0.109^{* *}$ & NS \\
\hline Level of lactoferrin in follicular fluid & 1.5 & 6.0 & $0.7(0.5-0.7)$ & 0.6 & 0.1 & $0.6(1-1)$ & $0.636^{* *}$ & NS \\
\hline Number of retrieved oocytes & 11.5 & 6.6 & $12(7-14)$ & 13.5 & 5.6 & $14(8-19)$ & $0.136^{* *}$ & NS \\
\hline Number of mature oocytes & 6.6 & 4.4 & $6.5(3.5-9)$ & 7.7 & 3.0 & $8(6-10)$ & 0.173 & NS \\
\hline Number of fertilized oocytes & 4.8 & 2.8 & $5(3-6)$ & 5.7 & 2.0 & $5(4-7)$ & 0.257 & NS \\
\hline Number of transferred embryo. & 2.3 & 1.0 & $2(2-3)$ & 2.5 & 0.6 & $3(2-3)$ & 0.429 & NS \\
\hline
\end{tabular}

${ }^{*}$ Student $\mathrm{t}$ test, ${ }^{* *}$ Mann-Whitney Test, ${ }^{* * *}$ Fisher exact.

Table 4. ROC Curve for prediction of biochemical pregnancy from Level of Lactoferrin in follicular fluid.

\begin{tabular}{cccccccc}
\hline Cutoff & AUC (CI) & Sensitivity & Specificity & + PV & - PV & P & Sig \\
\hline$\geq 0.66$ & $0.513(0.364$ to 0.66$)$ & 45.00 & 65.12 & 37.5 & 71.8 & 0.86 & NS \\
\hline
\end{tabular}

Table 5. Correlations between Level of lactoferrin in follicular fluid with number of retrieved, mature and fertilized oocyte.

\begin{tabular}{ccccc}
\hline & & $\begin{array}{c}\text { Number of } \\
\text { Retrieved oocytes }\end{array}$ & $\begin{array}{c}\text { Number of } \\
\text { mature oocytes }\end{array}$ & $\begin{array}{c}\text { Number of } \\
\text { fertilized oocytes }\end{array}$ \\
\hline lactoferrin in & $\mathrm{r}_{\mathrm{s}}^{*}$ & 0.051 & 0.101 & 0.071 \\
follicular fluid & $\mathrm{P}$ & 0.690 & 0.431 & 0.581 \\
& Significance & Non-significant & Non-significant & Non-significant \\
\hline
\end{tabular}

${ }^{\star}$ Spearman correlation. 


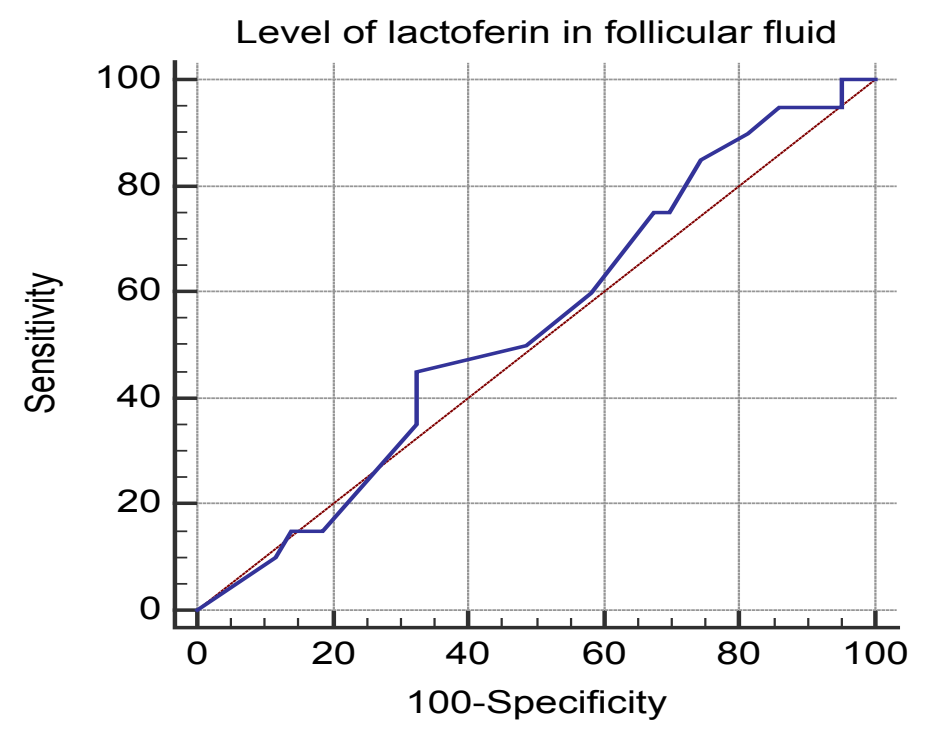

Figure 1. Receiver-operating characteristic (ROC) curve for prediction of clinical pregnancy using follicular fluid lactoferrin level. Dotted line represents reference line (line of random prediction).

The current study was a cross sectional study that included $64 \mathrm{FF}$ samples collected from 64 patients undergoing ICSI due to tubal, male, or unexplained infertility. The protocol used was flexible antagonist protocol. The study aimed to assess the relation between follicular fluid LF concentration and oocyte maturity, embryo quality, fertilization rate, and biochemical pregnancy rates.

There was no statistically significant difference between the group of women who achieved and the group of women who did not achieve biochemical pregnancy regarding: age, BMI, infertility cause or infertility duration.

The study revealed that there was no statistically significant difference between oocyte maturity, fertilization rate, embryo quality, or biochemical pregnancy rates and lactoferrin level in FF, in contrast to a study by (Yanaihara et al. (2007) [13] which was a retrospective study of 70 samples of FF at oocyte collection for 35 IVF patients for tubal factor infertility using modified natural cycle IVF protocol. The 70 samples were divided into fertilized group $(n=44)$ and unfertilized group $(n=26)$; the study reported that LF was present in detectable amounts in follicular fluid and the LF concentration was significantly higher in fertilized group compared to non-fertilized group; lactoferrin level in FF was also positively correlated with higher embryo quality. This study results might differ from our results due to measuring lactoferrin level from each follicle individually and not the mean lactoferrin level of FF of mature follicles collected from each patient which was done in the current study.

There was a limitation in the current study as the ICSI protocol depends on transfer of 2 to 3 embryos for each patient and not a single embryo transfer which could possibly give more precise results by accurately describing the relation between each follicular fluid lactoferrin level with its embryo quality and pregnancy rate. 
Study results also comes in contrast with (Zumoffen et al., 2013) [16] and (Zumoffen et al. 2015) [21] which studied the effect of LF on sperm capacitation and the dose-dependent effect of lactoferrin on gamete interaction, the study concluded that the presence of LF enhanced sperm capacitation in vitro by inducing dose dependent increase in induced acrosomal reaction and tyrosine phosphorylation of sperm proteins. This difference might be explained by different study methodology as the previous studies investigated the effect of lactoferrin on human gametes interaction in vitro and not in vivo.

The present study showed no significant correlation between lactoferrin concentration with age or BMI. However, (Moreno-Navarrete et al., 2009) [22] reported that circulating lactoferrin concentration was inversely associated with BMI, the Waist: hip ratio and the fasting triacylglycerol and glucose concentration and positively correlated with insulin sensitivity. They suggested that the LF production leads to decreased free lipopolysaccharide concentration and maintenance of an appropriate lipid profile.

The present study concluded that lactoferrin level in follicular fluid doesn't affect oocyte quality, fertilization rate, embryo quality or chemical pregnancy rate in ICSI cycles.

\section{Acknowledgements}

Patients participated in the study; Faculty of Medicine, Ain Shams University.

\section{Conflicts of Interest}

The authors declare no conflicts of interest regarding the publication of this paper.

\section{References}

[1] Revelli, A., DellePiane, L., Casano, S., Molinari, E., Massobrio, M. and Rinaudo, P. (2009) Follicular Fluid Content and Oocyte Quality: From Single Biochemical Markers to Metabolomics. Reproductive Biology and Endocrinology, 7, 40. https://doi.org/10.1186/1477-7827-7-40

[2] Fortune, J.E. (1994) Ovarian Follicular Growth and Development in Mammals. Biology of Reproduction, 50, 225-232. https://doi.org/10.1095/biolreprod50.2.225

[3] Nemet, K. and Simonovits, I. (1985) The Biological Role of Lactoferrin. Haematologia, 18, 3-12.

[4] Levay, P.F. and Viljoen, M. (1995) Lactoferrin: A General Review. Haematologica, 80, 252-267.

[5] Metz-Boutique, M.H., Jolles, J., Mazurier, J., Schoentgen, F., Legrand, D., Spik, G., Montreuil, J. and Jolles, P. (1984) Human Lactotransferrin: Amino Acid Sequence and Structural Comparisons with Other Transferrins. European Journal of Biochemistry, 145, 659-676. https://doi.org/10.1111/j.1432-1033.1984.tb08607.x

[6] Furmanskip, L.P., Fortuna, M.B., Swamy, C.V.B. and Das, M.R. (1989) Multiple Molecular Forms of Human Lactoferrin. Identification of a Class of Lactoferrins That Possess Ribonuclease Activity and Lack Iron-Binding Capacity. The Journal of Experimental Medicine, 170, 415-429. https://doi.org/10.1084/jem.170.2.415 
[7] Kelver, M.E., Kaul, A., Nowicki, B., Findley, W.E., Hutchens, T.W. and Nagamani, M. (1996) Estrogen Regulation of Lactoferrin Expression in Human Endometrium. American Journal of Reproductive Immunology, 36, 243-247. https://doi.org/10.1111/j.1600-0897.1996.tb00171.x

[8] Arici, A., Oral, E., Bukulmez, O., Buradagunta, S., Engin, O. and Olive, D.L. (1996) Interleukin-8 Expression and Modulation in Human Preovulatory Follicles and Ovarian Cells. Endocrinology, 137, 3762-3769. https://doi.org/10.1210/endo.137.9.8756544

[9] Belayet, H.M., Kanayama, N., Khatun, S., Asahina, T., Okada, Y., Kitamura, K., Terao, T., et al. (2000) Pharmacologic Doses of Interleukin 8 Suppositories Induce Follicular Maturation in Rabbits. Cytokine, 12, 361-367. https://doi.org/10.1006/cyto.1999.0553

[10] Ward, P.P., Mendoza, M.M., Saucedo-Cardenas, O., Teng, C.T. and Conneely, O.M. (1998) Restricted Spatiotemporal Expression of Lactoferrin during Murine Embryogenesis. In: Advances in Lactoferrin Research, Springer, Boston, 91-100. https://doi.org/10.1007/978-1-4757-9068-9_11

[11] Masson, P.L., Heremans, J.F. and Schonne, E. (1969) Lactoferrin, an Iron-Binbing Protein Ni Neutrophilic Leukocytes. Journal of Experimental Medicine, 130, 643-658. https://doi.org/10.1084/jem.130.3.643

[12] Gessler, P., Pretre, R., Hohl, V., Rousson, V., Fischer, J. and Dahinden, C. (2004) CXC-Chemokine Stimulation of Neutrophils Correlates with Plasma Levels of Myeloperoxidase and Lactoferrin and Contributes to Clinical Outcome after Pediatric Cardiac Surgery. Shock, 22, 513-520. https://doi.org/10.1097/01.shk.0000145939.54838.51

[13] Yanaihara, A., Mitsukawa, K., Iwasaki, S., Otsuki, K., Kawamura, T. and Okai, T. (2007) High Concentrations of Lactoferrin in the Follicular Fluid Correlate with Embryo Quality during in Vitro Fertilization Cycles. Fertility and Sterility, 87, 279-282. https://doi.org/10.1016/j.fertnstert.2006.06.025

[14] Tamura, T., Nozaki, A., Abe, K. I., Dansako, H., Naka, K., Ikeda, M., Kato, N., et al. (2005) cDNA Microarray Analysis of Lactoferrin Expression in Non-Neoplastic Human Hepatocyte PH5CH8 Cells. Biochimica et Biophysica Acta (BBA)-General Subjects, 1721, 73-80. https://doi.org/10.1016/j.bbagen.2004.10.003

[15] Sato, E. and Koide, S.S. (1984) A Factor from Bovine Granulosa Cells Preventing Oocyte Maturation. Differentiation, 26, 59-62. https://doi.org/10.1111/j.1432-0436.1984.tb01374.x

[16] Zumoffen, C.M., Gil, R., Caille, A.M., Morente, C., Munuce, M.J. and Ghersevich, S.A. (2013) A Protein Isolated from Human Oviductal Tissue in Vitro Secretion, Identified as Human Lactoferrin, Interacts with Spermatozoa and Oocytes and Modulates Gamete Interaction. Human Reproduction, 28, 1297-1308. https://doi.org/10.1093/humrep/det016

[17] Lin, Y.C., Chang, S.Y., Lan, K.C., Huang, H.W., Chang, C.Y., Tsai, M.Y., Huang, F.J., et al. (2003) Human Oocyte Maturity in Vivo Determines the Outcome of Blastocyst Development in Vitro. Journal of Assisted Reproduction and Genetics, 20, 506-512. https://doi.org/10.1023/B:JARG.0000013651.37866.0c

[18] Gardner, D.K., Surrey, E., Minjarez, D., Leitz, A., Stevens, J. and Schoolcraft, W.B. (2004) Single Blastocyst Transfer: A Prospective Randomized Trial. Fertility and Sterility, 81, 551-555. https://doi.org/10.1016/j.fertnstert.2003.07.023

[19] Revelli, A., Dolfin, E., Gennarelli, G., Lantieri, T., Massobrio, M., Holte, J.G. and Tur-Kaspa, I. (2008) Low-Dose Acetylsalicylic Acid plus Prednisolone as an Adju- 
vant Treatment in IVF: A Prospective, Randomized Study. Fertility and Sterility, 90, 1685-1691. https://doi.org/10.1016/j.fertnstert.2007.08.037

[20] Ward, P.P., Paz, E. and Conneely, O.M. (2005) Lactoferrin. Cellular and Molecular Life Sciences, 62, 2540. https://doi.org/10.1007/s00018-005-5369-8

[21] Zumoffen, C.M., Massa, E., Caille, A.M., Munuce, M.J. and Ghersevich, S.A. (2015) Effects of Lactoferrin, a Protein Present in the Female Reproductive Tract, on $\mathrm{Pa}$ rameters of Human Sperm Capacitation and Gamete Interaction. Andrology, 3, 1068-1075. https://doi.org/10.1111/andr.12093

[22] Moreno-Navarrete, J.M., Ortega, F.J., Bassols, J., Ricart, W. and Fernández-Real, J.M. (2009) Decreased Circulating Lactoferrin in Insulin Resistance and Altered Glucose Tolerance as a Possible Marker of Neutrophil Dysfunction in Type 2 Diabetes. The Journal of Clinical Endocrinology \& Metabolism, 94, 4036-4044.

https://doi.org/10.1210/jc.2009-0215 